\title{
Interdisciplinary peer review
}

\author{
Sustainability research is often interdisciplinary, presenting challenges and opportunities for authors, editors and \\ reviewers. Recognizing and contributing to its hard-won value is vital to unlocking the potential of sustainability \\ science and scholarship.
}

Sisis ustainability research often combines knowledge, data and methods from different fields. This breadth is a hallmark of sustainability research and contributes to its intellectual excitement and real-world relevance. But before that excitement and opportunity can be revealed, a study must pass the rubber and road of peer review. As authors, how many times have you dealt with this reality? As reviewers, how many times have you been asked to assess interdisciplinary work? Interestingly, sustainability research is still not as interdisciplinary as people might imagine (E. D. Schoolman et al. Sustain. Sci. 7, 67-80; 2012). The vagaries of peer review may play a role.

Peer review of interdisciplinary sustainability research has challenges and opportunities that are both generic and specific. Some are common to longestablished fields, such as medicine, and to relatively newer ones, such as science and technology studies. Common challenges, such as assessing interdisciplinary research holistically, are hardly trivial (see J. T. Klein Am. J. Prev. Med. 35, 116-123; 2008 and C. S. Wagner et al. J. Informetr. 7, 67-80; 2011). Others are probably more specific in part because sustainability research spans huge traditional divides, as among the natural sciences, social sciences and humanities. It even straddles more-professional fields, involving engineering, law, business and education, among others.

Authors must navigate a thicket of considerations in designing, doing and communicating interdisciplinary research. Early on, they must recognize what data, fields and methods to combine. What to combine often leads to who to convene; if researchers do not start out as interdisciplinarians or disciplinarians working together, they often end up so. This challenge extends to communicating the work. Differentiating disciplinary contributions may help reviewers and many readers, but this trades off with the value of weaving these contributions into a coherent narrative that perhaps better reflects the reality of the system or phenomena studied and the degree of interdisciplinary convergence involved (see the Comment by Irwin et al. in this issue).

Editors must navigate understanding and assessment while moderating a conversation of sorts between researchers and reviewers. Two challenges are recognizing interdisciplinary research and valuing it, both in the broad sense of acknowledging its inherent value and in the specific sense of assessing it relative to the probably few similar studies and perhaps numerous disciplinary ones on the same broad topic. Some studies will look at ecological variables as functions of social ones and vice versa, such as variation in soil organic carbon with different management regimes. Others will apply methods in one discipline to problems in another. Who should we recruit to evaluate such studies? How will we convince them to review? These are the trickiest questions. Most papers we send to review have reviewers from at least two disciplines, such as conservation biology and economics or civil engineering and public policy. Others have three. For papers integrating multiple disciplines for which most appropriate reviewers have relatively narrow research foci, we might also enlist an expert with knowledge of the broad context relevant to the research being reviewed. Deciding on what aspects need peer review is difficult, but enlisting reviewers - getting to 'yes' - can be daunting, especially for studies for which no one field is clearly central. Scholars are overextended regardless of discipline. A common response for interdisciplinary papers is that this is too far outside one's main research area. Editors do get it wrong sometimes, and we must do better at communicating the value of the research and the reviewing opportunity to those we ask. But if interdisciplinary research is to be properly assessed and valued, scholars must embrace this reality and recognize what they stand to gain - a peek into other fields, a glimpse of new applications in one's own, and the chance to shape those applications productively.
Finally, editors must digest, parse and combine reviews to assess interdisciplinary research both rigorously and holistically. This is a continual challenge, and we strive to keep improving.

Reviewers must weigh the pros and cons of accepting interdisciplinary reviews, stretch into unknown areas while focusing on their specialty, and, ideally, provide a final, broad assessment of the paper on its own terms. We recognize the multiple challenges involved and appreciate deeply the efforts of those who accept them. Today, some reviewers, like some authors, are themselves interdisciplinarians who may be especially busy given the additional demands of this career. Most are probably grounded primarily in one discipline, such as environmental engineering, anthropology or law, while doing research and perhaps teaching that reaches beyond.

All of us involved, from authors to editors to reviewers to the others playing key roles along the way, must grapple, however unconsciously, with one reality. Ultimately, doing and understanding interdisciplinary work involves knowing what to overlook. An interdisciplinary study of the reciprocal effects of economic incentives and fishing technology on reef biodiversity may never dive deeply enough into any of the contributing fields to satisfy some, but that is not a shortcoming. It may never inform textbook theory in ecology, economics or engineering. It need not, though what is textbook is itself contested and evolving. Knowing what to leave out and what to overlook is a hard-won and ever changing challenge. It is also a fulfilling one; let's embrace it.

Doing and assessing interdisciplinary sustainability research is hardly an easy path; some days there is no clear path at all. It is deeply gratifying and important, though. And as the poet Antonio Machado wrote, "Se hace camino al andar" - walking makes the road.

Published online: 16 July 2018 https://doi.org/10.1038/s41893-018-0111-3 